\title{
Universal Hysteresis Scaling for Incommensurate Magnetic Order in Dysprosium
}

\author{
Satoru Kobayashi* \\ NDE and Science Research Center, Faculty of Engineering, Iwate University, Ueda 4-3-5, Morioka 020-8551, Japan
}

(Received 12 December 2010; published 3 February 2011)

\begin{abstract}
We study the scaling properties of magnetic minor hysteresis loops in a polycrystalline dysprosium metal, varying temperature and magnetic-field amplitudes. We observe irreversibility-related hysteresis loss in the helical antiferromagnetic phase, which is related with remanent flux density as a power law with the same scaling exponent of $1.25 \pm 0.05$ as that in ferromagnetic materials. In contrast to hysteresis scalings in ferromagnets associated with $180^{\circ}$ Bloch walls, the observed law is governed by spiral walls which separate helical domains with oppositely rotating spins.
\end{abstract}

DOI: 10.1103/PhysRevLett.106.057207

PACS numbers: 75.60.Ej, 75.60.Ch

Magnetic domain wall, an interfacial region which separates domains with opposite magnetization, has been widely and extensively studied because of their technological and engineering applications such as future spintronics device and fundamental physical importances [1,2]. Their static and dynamical motion has led to diverse universal and scaling phenomena in Barkhausen avalanches [3-5], the field-velocity characteristics [6], and hysteresis behavior [7-12]. In particular, magnetic hysteresis loops, which reflect irreversible wall motion under pinning fields, have been shown to exhibit scaling power laws in their hysteresis parameters. For instance, for thin ferromagnetic films, dynamical hysteresis loop area $A$ has been shown to relate with applied field strength $H$, frequency $\Omega$, and temperature $T$ as $A \propto H^{\alpha} \Omega^{\beta} T^{-\gamma}$ or $A \propto(d H / d t)^{\alpha}$ with scaling exponents $\alpha, \beta$, and $\gamma$ [7-9]. On the other hand, for bulk ferromagnets, the empirical Steinmetz law, which relates static loop area, i.e., hysteresis loss $W_{F}^{*}$ and maximum flux-density $B_{a}^{*}$ as $W_{F}^{*} \propto\left(B_{a}^{*}\right)^{1.6}$, has been long known [10-12]. Nevertheless, all scaling laws are generally governed by the motion of $180^{\circ}$ domain walls and have only been observed in ferromagnetic materials.

In this Letter, we report the first observation of a scaling power law of magnetic hysteresis loops in a helical incommensurate magnetic phase with spins rotated from atomic layer to layer and with no net spontaneous magnetization. The observed law is governed by motion of spiral walls which separate helical domains with spins rotating clockwise or anticlockwise. We have focused on a bulk sample of heavy rare-earth metal Dy which shows a rich magnetic-temperature phase diagram with various types of magnetic ordering [13-17]. Below a Néel temperature of $T_{N} \sim 180 \mathrm{~K}$, Dy exhibits a helical antiferromagnetic (HAM) structure where magnetic moments confined on the hexagonal basal planes rotate from basal plane to basal plane. With decreasing temperature, the turn angle between the adjacent planes decreases from about $43.2^{\circ}$ to $26.5^{\circ}$ and the magnetic phase transition toward the ferromagnetic (FM) phase takes place at $T_{c} \sim 90 \mathrm{~K}$ below which all the magnetic moments align ferromagnetically along the $a$ axis. These magnetically ordered phases are stabilized as a consequence of magneto-crystalline anisotropy energy, magnetostriction energy, and long-range competing exchange interactions [14]. Earlier works focusing on the nature of the first-order HAM-FM phase transition revealed an anomalous increase in ultrasonic attenuation $[18,19]$ and ac magnetic susceptibility $[20,21]$ in a wide temperature range above $T_{c}$. This anomaly was only observed on heating from the FM phase through $T_{c}$ and could be due to the nucleation of the large number of spiral walls in the HAM phase.

A toroidal sample of polycrystalline Dy was cut from an ingot with a purity of $99.9 \%$ by an electric discharge machine and was then polished to eliminate oxidation layers; external and internal diameters of 9 and $7 \mathrm{~mm}$, respectively, and thickness of $2.7 \mathrm{~mm}$. Flux-density $(B)$ field $(H)$ loops were measured with a conventional fluxmetric method [22,23]. A 140-turn exciting and 100-turn detecting coils of copper wires were wound around the sample to generate a cyclic magnetic field in the circumferential direction and pick up the induced voltage due to magnetization, respectively. The sampling rate was $2000 \mathrm{~Hz}$. $B-H$ loops with various field amplitudes $H_{a}$ up to $22 \mathrm{kA} / \mathrm{m}$ were measured by step-by-step increasing $H_{a}$, keeping the speed of applied field $d H / d t$. Before the measurements, the sample was demagnetized with a decaying alternating magnetic field with $4 \mathrm{~Hz}$. Various values of $d H / d t$ in the range of $8-64 \mathrm{kA} / \mathrm{m} / \mathrm{s}$ were tested. Since the results were weakly dependent on $d H / d t$, the eddy current effect was not important and the results for $d H / d t=64 \mathrm{kA} / \mathrm{m} / \mathrm{s}$ will be given in this study. The sample was placed in a He-gas closed-cycle refrigerator and cooled down to $5 \mathrm{~K}$. Measurements were performed at intervals of $2 \mathrm{~K}$ on heating from $T=5 \mathrm{~K}$ or cooling from $T=200 \mathrm{~K}$ in the paramagnetic (PM) phase above $T_{N}$. The temperature was stabilized at each measuring temperature within $0.05 \mathrm{~K}$.

Figs. 1(a) and 1(b) show a set of $B-H$ loops with various field amplitude $H_{a}$, taken at various temperatures on heating and cooling, respectively. In Fig. 2(a), the temperature 

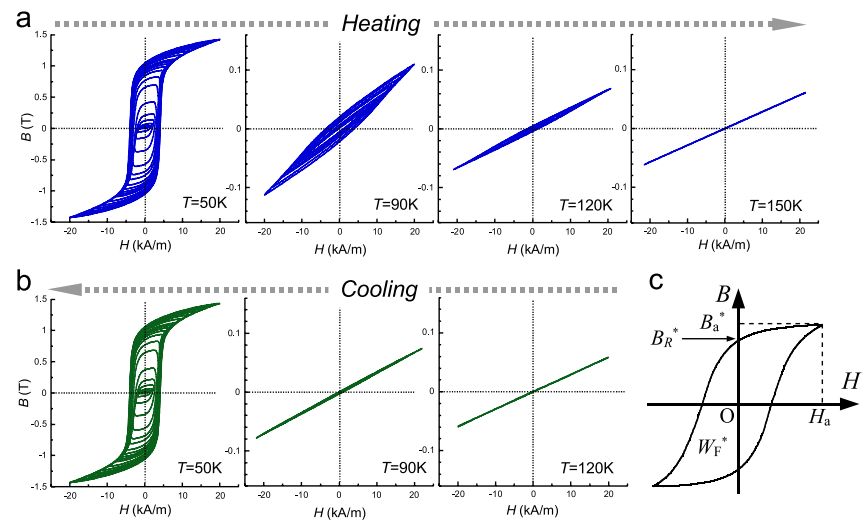

FIG. 1 (color online). $\quad B$ - $H$ loops with various field amplitudes $H_{a}$, taken at different temperatures on (a) heating and (b) cooling. (c) Parameters of a $B-H$ loop; $B_{a}^{*}, B_{R}^{*}$, and $W_{F}^{*}$ are maximum flux density, remanent flux density, and hysteresis loss, respectively.

dependence of minor-loop initial permeability $\mu_{m}$ is shown. Here, $\mu_{m}$ was obtained from a linear part of $B_{a}^{*}-H_{a}$ curves for $B_{a}^{*}$ below $\sim 2 \times 10^{-3} \mathrm{~T}$ as shown in the inset in Fig. 2(a); $T_{N}=175 \mathrm{~K}$, and $T_{c}=87 \mathrm{~K}$ and $85 \mathrm{~K}$ for heating and cooling, respectively. In the FM phase, a hysteresis behavior typical for ferromagnetic materials was observed [Fig. 1(a): $T=50 \mathrm{~K}$ ]. With increasing temperature, $B_{a}^{*}$ gradually decreases accompanying the narrowing of the loop width and the ferromagnetic property disappears with the onset of the HAM phase at $T_{c}$. However, the hysteresis behavior was clearly observed even above $T_{c}$ [Fig. 1(a): $T=90$ and $120 \mathrm{~K}$ ] and persists up to a rather high temperature of $\sim 140 \mathrm{~K}$. More interestingly, such hysteresis behavior in the HAM phase was not observed on cooling [Fig. 1(b): $T=90$ and $120 \mathrm{~K}$ ], though almost the same results were obtained for the FM ordering below $T_{c}$. This is reflected in the temperature dependence of $\mu_{m}$ shown in Fig. 2(a), where $\mu_{m}$ obtained on heating shows a larger value in the HAM phase up to $T \sim 140 \mathrm{~K}$. Such anomalous temperature hysteresis was also reported in ultrasonic measurements $[18,19]$ and ac susceptibility measurements [20,21].

In order to investigate whether existing scaling laws hold true below and above $T_{c}$, parameters of $B$ - $H$ loops were defined as denoted in Fig. 1(c). In this work, we focused on two scaling power laws of $B-H$ loops between $W_{F}^{*}$ and $B_{a}^{*}$ and between $W_{F}^{*}$ and $B_{R}^{*}$, where $B_{R}^{*}$ is remanent flux density; $W_{F}^{*}=W_{F}^{0}\left(B_{a}^{*} / B_{s}\right)^{n_{F}}$ and $W_{F}^{*}=W_{m}^{0}\left(B_{R}^{*} / B_{s}\right)^{n_{m}}$, where $n_{F}$ and $n_{m}$ are a power law exponent, $W_{F}^{0}$ and $W_{m}^{0}$ are a coefficient being sensitive to materials intrinsic properties, $B_{s}$ is normalization constant and was assumed to be saturation magnetization of $3.8 \mathrm{~T}$, which corresponds to full magnetic moment of Dy atom, $10.3 \mu_{B}$ /atom [14]. The former law is the well-known Steinmetz law [10], whereas the latter is the recently observed one which only reflects the irreversible domain wall motion even

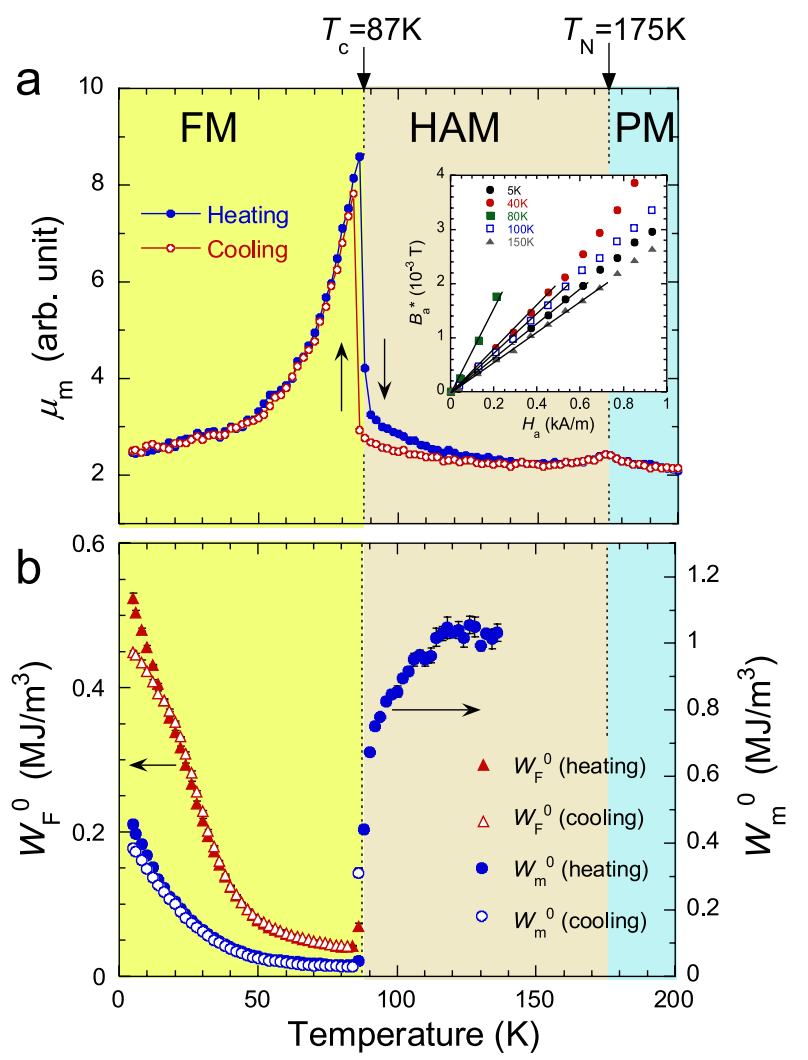

FIG. 2 (color online). (a) Minor-loop initial permeability $\mu_{m}$ and (b) coefficients of scaling power laws, $W_{F}^{0}$ and $W_{m}^{0}$ as a function of temperature. The solid and open circles represent the data taken on heating and cooling, respectively. The inset in (a) shows $B_{a}^{*}$ as a function of $H_{a}$ at several temperatures, taken on heating. The triangles and circles in (b) denote $W_{F}^{0}$ and $W_{m}^{0}$, respectively.

under the influence of reversible mechanism due to domain wall motion and domain rotation [22,23].

Figure 3(a) shows a double logarithmic plot of $W_{F}^{*}-B_{a}^{*}$ curves, taken at various temperatures on heating. In the FM phase, a linear part is seen for $B_{a}^{*}$ above $\sim 0.2 \mathrm{~T}$, indicating the existence of a power law between $W_{F}^{*}$ and $B_{a}^{*}$. Leastsquares fits yielded a power law exponent of $n_{F}=1.43 \pm$ 0.05 for both heating and cooling data, being consistent with values of soft ferromagnetic materials $[10,23]$. On the other hand, in the HAM phase above $T_{c}$, the $W_{F}^{*}-B_{a}^{*}$ curves show a steep slope with $n_{F} \sim 3$ in the low $B_{a}^{*}$ regime, indicating the dominant contribution of domain rotation in this $B_{a}^{*}$ range in the HAM phase. In order to extract irreversibility-related properties from the observed $B-H$ loops, we examined the relation between $W_{F}^{*}$ and $B_{R}^{*}$. The $W_{F}^{*}-B_{R}^{*}$ relation shows a linearity in a wide $B_{R}^{*}$ range below $\sim 0.6 \mathrm{~T}$ in a double logarithmic plot in the FM phase as shown in Fig. 3(b). However, this linearity persists up to $\sim 140 \mathrm{~K}$ well above $T_{c}$, keeping the slope almost constant as shown in the inset in Fig. 3(b). Least-squares fits yielded a very similar value of $n_{m}$ for both phases and 

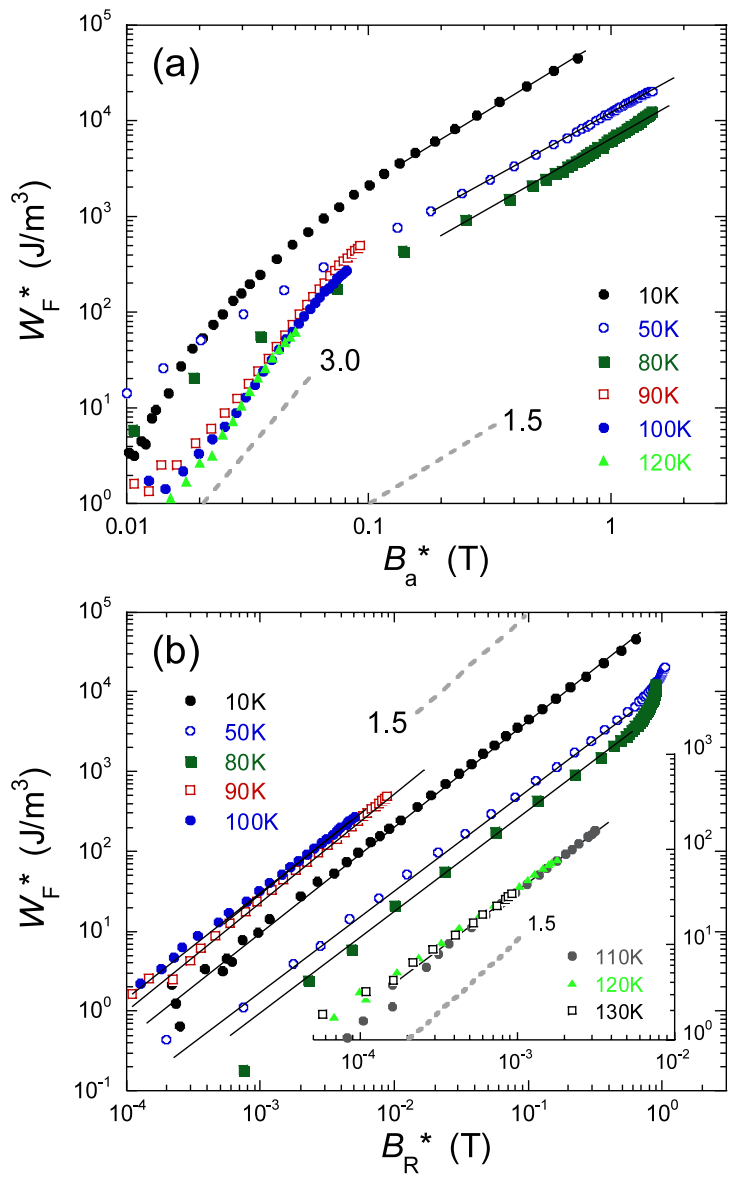

FIG. 3 (color online). (a) Relationship between $W_{F}^{*}$ and $B_{a}^{*}$ at different temperatures on heating, plotted on a double logarithmic scale. Slopes of 1.5 and 3.0 are given. The solid lines through the data denote least-squares fits. An increase of a slope in a low $B_{a}^{*}$ range in the FM phase at low temperatures is due to an increasing contribution of reversible Bloch wall motion and domain rotation [23]. (b) Relationship between $W_{F}^{*}$ and $B_{R}^{*}$ at different temperatures on heating, plotted on a double logarithmic scale. The inset shows $W_{F}^{*}-B_{R}^{*}$ curves at higher temperatures above $T_{c}$.

$n_{m}=1.25 \pm 0.05$, being in good agreement with 1.3-1.4 obtained for soft ferromagnetic materials [23]. These observations indicate that irreversible motion of domain walls plays a significant role for hysteresis behaviors in both phases. To our best knowledge, this is the first experimental observation of a hysteresis scaling law in the incommensurate magnetic phase.

Figure 2(b) shows a coefficient $W_{F}^{0}$ and $W_{m}^{0}$ as a function of temperature. In the FM phase both coefficients monotonically decrease with increasing temperature. With the onset of the HAM ordering at $T_{c}, W_{m}^{0}$ exhibits a sudden increase and continuously increases up to $T \sim 140 \mathrm{~K}$, above which a hysteresis behavior could not be observed with the accuracy of our measurements. The data obtained on cooling show a quite similar trend as those on heating in the FM phase.

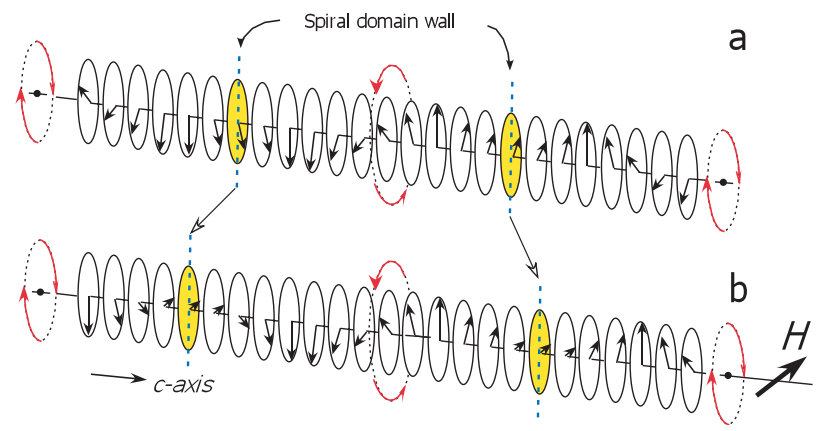

FIG. 4 (color online). (a) Schematic drawing of the spiral walls (blue dotted lines) in zero field in the HAM phase. The arrows in the circle represent magnetic moments, which rotate on the hexagonal basal plane. The spiral walls separate helical antiferromagnetic domains with magnetic moments rotating in an opposite way. (b) Schematic drawing of the spiral walls in a magnetic field on the basal plane. Magnetic moments on spiral walls tend to direct along the field direction, associated with modification in the domain structure.

Below $T_{c}$, magnetic moments are confined along the $a$ axis and $180^{\circ}$ Bloch walls in which magnetic moments rotate on the hexagonal basal plane separate antiphase FM domains. Irreversible movement of the Bloch walls, associated with the pinning by such as lattice defects would yield the hysteretic behavior of $B-H$ loops, yielding scaling power laws as observed in soft ferromagnetic materials [23]. As the temperature increases from below $T_{c}$, the HAM structure with incommensurate wave number along the $c$ axis is formed at $T_{c}$. There would exist two processes to form HAM structure; (i) other ferromagnetic domains are nucleated within ferromagnetic domains and for each domain magnetic moment changes its orientation from layer to layer to form the HAM structure; (ii) Bloch walls which exist in the FM phase simply widen to form the HAM structure [18]. Both processes will produce a large number of spiral domain walls separating HAM domains whose magnetic moments rotate on the basal plane in an opposite way with respect to the $c$ axis, i.e., clockwise or anticlockwise, as shown in Fig. 4(a) [18-21].

The spiral domain walls behave as thin ferromagnetic layers and displace in the presence of applied field in the basal plane, associated with some modifications of local magnetic structure as shown in Fig. 4(b). These spiral walls would give a significant contribution to net magnetization in the HAM phase, besides magnetization arising from a distortion of the HAM structure in the domains. The irreversibility of spiral wall motion under pinning fields would lead to a hysteresis loss in the HAM phase, resulting a scaling power law between $W_{F}^{*}$ and $B_{R}^{*}$ as observed in the FM phase.

Here, we note a possible coexistence of the FM and HAM phases around $T_{c}$. As revealed by recent x-ray powder diffraction measurements [17], both FM and HAM phases coexist at around $T_{c}$ both on heating and 
cooling over a $\sim 10 \mathrm{~K}$ wide region for polycrystalline Dy, which is larger than $\sim 4 \mathrm{~K}$ for a single crystal sample [15]; such a magnetically heterogeneous state persists up to $\sim 100 \mathrm{~K}$ and a single HAM state is achieved above the temperature in zero field. Therefore, we consider that the observed scaling law above $\sim 100 \mathrm{~K}$ is essentially related to irreversibility of spiral domain walls, whereas in an intermediate temperature region between $T_{c}$ and $\sim 100 \mathrm{~K}$, spiral domain walls between HAM domains, Bloch walls between FM domains, and boundaries between FM and HAM domains may contribute to the scaling law; basically, the scaling exponent $n_{m}$ is not affected by the heterogeneity [23], though a minor-loop coefficient $W_{m}^{0}$ may be slightly modified. It is also noted that though such a heterogeneous temperature region shifts toward a higher temperature in a magnetic field [17], our maximum measurement field of $22 \mathrm{kA} / \mathrm{m}$ is small enough to reach the region; for instance, a magnetic field of $240 \mathrm{kA} / \mathrm{m}$ is necessary at $T=110 \mathrm{~K}$.

Finally, we remark the behavior of $W_{F}^{0}$ and $W_{m}^{0}$, which are a sensitive indicator of pinning field [23]. For Dy there exists very large sixfold basal anisotropy in the FM phase and a width of Bloch wall is less than 20 atomic layers, which is much smaller than a few hundred atomic layers for soft ferromagnetic materials. Therefore, the energy of Bloch walls depends on the center position with respect to the atomic lattice, giving rise to another pinning mechanism due to the Peierls potential $[24,25]$ in addition to usual wall pinning due to lattice defects. The decrease of the basal anisotropy with increasing temperature [26] reduces the pinning energy, resulting in a decrease in $W_{F}^{0}$ and $W_{m}^{0}$ in the FM phase. In the HAM phase, the contribution of the anisotropy is very small and the spiral wall energy is mainly dominated by exchange energy. According to a simple calculation taking account of the temperature variation of helical turn angle, the number of magnetic moments which comprise a spiral domain wall decreases with temperature and the wall energy maximizes around $120-130 \mathrm{~K}$ [27]. This implies that the energy to overcome the pinning potential is high at around this temperature, being consistent with the high value of $W_{m}^{0}$ at $T \sim 130 \mathrm{~K}$.

The author thanks S. Takahashi for discussions. This research was partly supported by president's research fund of Iwate University and a Grant-in-Aid for Scientific Research (S), Grant No. 14102034, from the Ministry of Education, Culture, Sports, Science and Technology of Japan. *koba@iwate-u.ac.jp

[1] J. P. Liu, E. Fullerton, O. Gutfleisch, and D. J. Sellmyer, Nanoscale Magnetic Materials and Applications (Springer, New York, 2009).

[2] G. Bertotti, Hysteresis in Magnetism (Academic Press, San Diego, 1998).

[3] K.-S. Ryu, H. Akinaga, and S.-C. Shin, Nature Phys. 3, 547 (2007).

[4] G. Durin and S. Zapperi, Phys. Rev. Lett. 84, 4705 (2000).

[5] E. Puppin, Phys. Rev. Lett. 84, 5415 (2000).

[6] M. Yamanouchi, J. Ieda, F. Matsukura, S. E. Barnes, S. Maekawa, and H. Ohno, Science 317, 1726 (2007).

[7] J.S. Suen and J.L. Erskine, Phys. Rev. Lett. 78, 3567 (1997).

[8] C. Nistor, E. Faraggi, and J. L. Erskine, Phys. Rev. B 72, 014404 (2005).

[9] F. Colaiori, G. Durin, and S. Zapperi, Phys. Rev. Lett. 97, 257203 (2006).

[10] C. P. Steinmetz, Proc. IEEE 72, 197 (1984).

[11] R. M. Bozorth, Ferromagnetism (IEEE, New York 1978), and references therein.

[12] F. J. G. Landgraf, M. Emura, and M. F. de Campos, J. Magn. Magn. Mater. 320, e531 (2008).

[13] W. C. Koehler, J. Appl. Phys. 36, 1078 (1965).

[14] S. Legvold, in Ferromagnetic Materials, edited by E. P. Wohlfarth (North-Holland Physics Publishing, Amsterdam, 1986), p. 183.

[15] V. V. Vorob'ev, M. Y. Krupotkin, and V. A. Finkel, Sov. Phys. JETP 61, 1056 (1985).

[16] A.S. Chernyshov, A. O. Tsokol, A. M. Tishin, K. A. Gschneidner, Jr., and V.K. Pecharsky, Phys. Rev. B 71, 184410 (2005).

[17] A. S. Chernyshov, Y. Mudryk, V. K. Pecharsky, and K. A. Gschneidner, Jr., Phys. Rev. B 77, 094132 (2008).

[18] S. B. Palmer, J. Phys. F 5, 2370 (1975).

[19] G. N. Blackie and S. B. Palmer, Portgal. Phys. 11, 23 (1980).

[20] T. J. McKenna, S. J. Campbell, D. H. Chaplin, and G. V. H. Wilson, J. Magn. Magn. Mater. 20, 207 (1980).

[21] A. Del Moral and E. W. Lee, J. Phys. F 4, 280 (1974).

[22] S. Kobayashi, A. Saito, S. Takahashi, Y. Kamada, and H. Kikuchi, Appl. Phys. Lett. 92, 182508 (2008).

[23] S. Kobayashi, S. Takahashi, T. Shishido, Y. Kamada, and H. Kikuchi, J. Appl. Phys. 107, 023908 (2010).

[24] R. Herz and H. Kronmüller, J. Magn. Magn. Mater. 4, 36 (1977).

[25] T. Egami and C.D. Graham Jr., J. Appl. Phys. 42, 1299 (1971).

[26] D. J. Martin and J. J. Rhyne, J. Phys. C 10, 4123 (1977).

[27] T. J. McKenna, S. J. Campbell, D. H. Chaplin, and G. V. H. Wilson, J. Phys. Condens. Matter 3, 1855 (1991). 